\title{
Frontières
}

\section{Nouvelles parutions - Études sur la mort}

\section{Ngoc-Than Phan Nguyen}

Volume 18, numéro 1, automne 2005

URI : https://id.erudit.org/iderudit/1074330ar

DOI : https://doi.org/10.7202/1074330ar

Aller au sommaire du numéro

Éditeur(s)

Université du Québec à Montréal

ISSN

1180-3479 (imprimé)

1916-0976 (numérique)

Découvrir la revue

Citer ce document

Nguyen, N.-T. P. (2005). Nouvelles parutions - Études sur la mort. Frontières, 18(1), 81-82. https://doi.org/10.7202/1074330ar d'utilisation que vous pouvez consulter en ligne.

https://apropos.erudit.org/fr/usagers/politique-dutilisation/ 


\section{NOUVELLES PARUTIONS - ÉTUDES SUR LA MORT}

Ngoc-Than Phan Nguyen

ALTOUNIAN, Janine (2005). L'intraduisible: deuil, mémoire, transmission, Paris, Dunod.

ALDRIDGE, David (2005). Music Therapy in Palliative Care: New Voices, Vancouver, UBC Press.

ALDRIDGE, David (2005). Suicide: The Tragedy of Hopelessness, Vancouver, UBC Press.

ANCELIN SCHÜTZENBERGER, Anne et BISSONE JEUFROY, Evelyne (2005). Sortir du deuil: surmonter son chagrin et réapprendre à vivre, Paris, Payot.

ANGENAULT, Marcel (2005). Vivre avec le cancer: les acteurs du combat, Paris, Archipel.

APPLETON, Michael (2005). Good End: End-Of-Life Concerns and Conversations about Hospice and Palliative Care, Tucson, Arizona, Hats Off Books.

BATTIN, Margaret Pabst (2005). Ending Life: Ethics and the Way We Die, Londres, Oxford University Press.

BEAULIEU, Marie-Bernadette et al. (2005). La personne âgée: rôle de l'aide-soignant en institution et à domicile, $3^{\mathrm{e}}$ éd., Issy-les-Moulineaux (Hauts-de-Seine), Masson.

BINOCHE, Thierry et Martineau, CAROLINE (2005). Guide pratique du traitement des douleurs, Issy-les-Moulineaux (Hauts-de-Seine), Masson, coll. «Médiguides».

CAVEY, Michel (2005). L'euthanasie: pour un débat dans la dignité, Paris, L'Harmattan.

CENTRE D'EXPERTISE COLLECTIVE DE L'INSERM, Paris (2005). Suicide: autopsie psychologique, outil de recherche en prévention. Paris, Inserm, coll. "Expertise collective».

CHANU, Antoinette (2005). Euthanasie: vous avez demandé une loi?, Paris, J. Lyon, coll. «Santé minute».

CLERGET, Joël (dir.) et al. (2005). Bébé est mort: expériences, rites et célébrations, RamonvilleSaint-Agne (Haute-Garonne), Erès, coll. «Mille et un bébés. Drames et aléas de la vie des bébés".

COLLET, Victor (2005). Canicule 2003: origines sociales et ressorts contemporains $d^{\prime}$ 'une mort solitaire: les personnes seules du cimetière de Thiais, Paris, L'Harmattan, coll. "Questions contemporaines".

CONSEIL INTERNATIONAL DE LA CHASSE (2005). Le chasseur et la mort, Paris, la Table ronde.

DERVILLE, Tugdual (2005). Le bonheur blessé: avortement, eugénisme et euthanasie en question, Tours, CLD.

DOKA, Kenneth J. (2005). Living with Grief: Ethical Dilemmas at the End of Life, Washinton, D.C., Hospice Foundation of America.

DOYLE, Derek (2005). Oxford Textbook of Palliative Medicine, $3^{\mathrm{e}}$ éd. , Londres, Oxford University Press.

DYREGROV, Atle (2005). Grief in Children, Vancouver, UBC Press.
FALCON, Georgina (2005). En traversant le pont, Montréal, Mediaspaul, coll. «Vivre plus».

FORT, Josette-Alberte (2005). Naissance et fantasme de mort, Paris, L'Harmattan, coll. «Sexualité humaine».

FOUCAULT, Claudette et MONGEAU, Suzanne (2004). L'art de soigner en soins palliatifs: perspectives infirmières, $2^{\mathrm{e}}$ éd., Montréal, Presses de I'Université de Montréal, coll. «Paramètres».

FROUCHT-HIRSCH, Sylvie (2005). Le temps d'un cancer: chroniques d'un médecin malade, Paris, Vuibert, coll. "Espace éthique».

GOLDBERGER, Corine (2005). Quand la mort sépare un jeune couple: le veuvage précoce, Paris, Albin Michel.

GORSUCH, Neil M. (2005). The Future of Assisted Suicide and Euthanasia in America, Princeton, Princeton University Press.

GUDIN, Claude (2005). Une histoire naturelle de la mort, Lausanne (Suisse), l'Âge d'homme, coll. "Hypothèse».

HAUMONT, Marie-Claude (2005). Le chemin de I'acceptation: voie de la guérison, de la réussite, du bien-être, Cholet (Maine-et-Loire), Les 2 encres, coll. «Comment réussir au quotidien».

HAWTON, Keith (2005). Prevention and Treatment of Suicidal Behaviour: From Science to Practice, Londres, Oxford University Press.

HIRSCH, Emmanuel (dir.) (2005). Face aux fins de vie et à la mort: éthique et pratiques professionnelles au cœur du débat, Paris, Vuibert, coll. «Espace éthique».

HOLDEREGGER, Adrian (2005), Le suicide: le conflit entre la vie et la mort, Paris, Cerf.

HOLMES, Ronald M. et HOLMES, Stephen T. (2005). Suicide: Theory, Practice and Investigation, Thousand Oaks, Cal. , Sage Publications.

HOROWITZ, Elisabeth (2005). Les fantômes du passé: comment les deuils familiaux influencent notre vie, Paris, Dervy, coll. "Chemin de l'harmonie». HOSIER, Helen Kooiman (2005). An Eclipse of the Soul: A Christian Resource on Dealing with Suicide, Toronto, R.G. Mitchell.

HUGHES, Selwyn (2005). Le bien-être et la guérison: guide pratique, Paris, Empreinte temps présent. JAROSZ, Maria (2005). Suicides, traduit du polonais par Wanda Gaignebet, Paris, L'Harmattan, coll. "Logiques sociales. Déviance».

KÉBERS, Claire (2005). Soins curatifs, soins palliatifs: leurs différences et leur complémentarité, Bruxelles, Racine.

KHOSROKHAVAR, Farhad (2005). Suicide Bombers: Allah's New Martyrs, Londres, Pluto Press.

KUEBLER, Kim (2005). Palliative Practices, Toronto, Mosby.

KUEHN, Eileen (2005). After Suicide: Living with the Questions, Markham, Ont., Fitzhenry \& Whiteside.

LAMUNIÈRE, Marie-Claire (2005). Nous ne vieillirons pas ensemble: témoignage, Vevey (Suisse), Aire.

LEDOGAR, Denis (2005). Seul l'amour fracasse les tombeaux, Paris, Presses de la Renaissance, coll. "Spiritualité et témoignage».
LEGUAY, Catherine (2005). Respecter la vie, disposer de sa mort!: pour une loi Vincent Humbert, Paris, L'Harmattan, coll. "Questions contemporaines".

LETHIERRY, Hugues (dir.) (2005). Parler de la mort... et de la vie: un tabou dans l'éducation?, Paris, Nathan, coll. «Repères pédagogiques».

LÉVESQUE, Nicolas (2005). Le deuil impossible nécessaire, Québec, Nota Bene.

MAISON MONBOURQUETTE (2005). Répertoire des ressources en suivi de deuil au Québec, Montréal, Maison Monbourquette.

MASON, Christina (2005). Journeys Into Palliative Care: The Dynamics of Groups in Therapeutic, Educational and Corporate Settings, Vancouver, UBC Press.

MATTHEEUWS, Alain (2005). Accompagner la vie dans son dernier moment, Saint-Maur (Val-deMarne), Parole et silence, coll. «Cahiers de l'École cathédrale».

MEYNIEL, Dominique (2005). Le couloir des urgences, Paris, LGF, coll. "Le livre de poche».

MORDANT, Claude (dir.) et al. (2005). Les pratiques funéraires à l'âge du bronze en France: actes de la table ronde de Sens en Bourgogne (Yonne), 10-12 juin 1998, Paris, Éd. du CTHS, coll. «Documents préhistoriques".

MORON, Pierre (2005). Le suicide, $7^{e}$ éd., Paris, Presses universitaires de France, coll. "Que sais-je?». NAKAYA, Andrea C. (2005). Terminal IIIness: Opposing Viewpoints, San Diego, Greenhaven Press.

NEIMEYER, Robert A. (2005). Lessons of Loss: A Guide to Coping, New York, Routledge.

PAPE, Robert (2005). Dying to Win: The Strategic Logic of Suicide Terrorism, New York, Random House.

PEACOCK, Judith (2005). Teen Suicide, Toronto, Fitzhenry \& Whiteside.

PÉCHERY-CONDAT, Florence (2005). Ton combat pour la vie: traverser le cancer à 17 ans, Saint-Maur (Val-de-Marne), Parole et silence.

PERNAUT, Nathalie (2005). Le cancer en face: donnons toutes ses chances à la vie, Neuilly-sur-Seine (Hauts-de-Seine), M. Lafon.

PERROT, Serge (2005). Douleur aiguë, douleur chronique, soins palliatifs, Paris, MED-LINE, coll. «Mémo-flash MED-LINE».

PIETTE, Albert (2005). Le temps du deuil, Paris, Éd. de l'Atelier.

PINQUE, Georges (2005). Cancer: thérapies d'aujourd'hui et de demain, Paris, G. Trédaniel.

PRIEUR, Cécile (dir.) et al. (2005). La peine de mort, Paris, Librio.

RACHET, Guy (2005). Les chemins de l'autre monde: apprendre à mourir, Paris, Bartillat.

RENAULT, Danièle (2005). Travail de deuil, trajet de vie et transition psychosociale: les enjeux biocognitifs d'un accompagnement, Paris, Mare \& Martin.

SACKET, Joyce (2005). Goodbye Jeanine: A Mother's Faith Journey after Her Daughter's Suicide, Toronto, R.G. Mitchell.

SAMOUEL, Valérie et SATE, Pierre (2005). 100 réponses sur le suicide des adolescents, Paris, Tournon, coll. « 100 réponses sur». 
SCHOTSMANS, Paul (2005). Euthanasia and Palliative Care in the Low Countries, Leuven, Peeters.

SCOTT, Bill (2005). Suicide: It Isn't What You Think, Baltimore, Publish America.

SITTSER, Gerald Lawson (2005). A Grace Disguised: How the Soul Grows Through Loss, Toronto, HarperCollins.

SMITH, Wesley J. (2005). Forced Exit: Euthanasia, Assisted Suicide and the New Duty to Die, San Francisco, Encounter Books.

SOCIÉTÉ FRANÇAISE D'ACCOMPAGNEMENT ET DE SOINS PALLIATIFS. COLLÈGE SOINS INFIRMIERS, Île-de-France (2005). L'infirmier(e) et les soins palliatifs: prendre soin: éthique et pratiques, Issy-les-Moulineaux (Hauts-de-Seine), Masson, coll. "Savoir et pratique infirmière».
SONTAG, Susan (2005). La maladie comme métaphore, traduit de l'américain par Marie-France de Paloméra, Paris, Bourgois.

SONTAG, Susan (2005). Le sida et ses métaphores, traduit de l'américain par Brice Matthieussent, Paris, Bourgois.

TULLOCH, Gail (2005). Euthanasia - Choice and Death, Vancouver, UBC Press.

URBAIN, Jean-Didier (2005). L'archipel des morts, nouv. éd., Paris, Payot.

VALENTINE, Bill (2005). A Season of Grief, New York, Harrington Park Press.

VEILLE, Frédéric (2005). Vincent Humbert, de I'enfer au paradis blanc, Grainville (Eure), City.

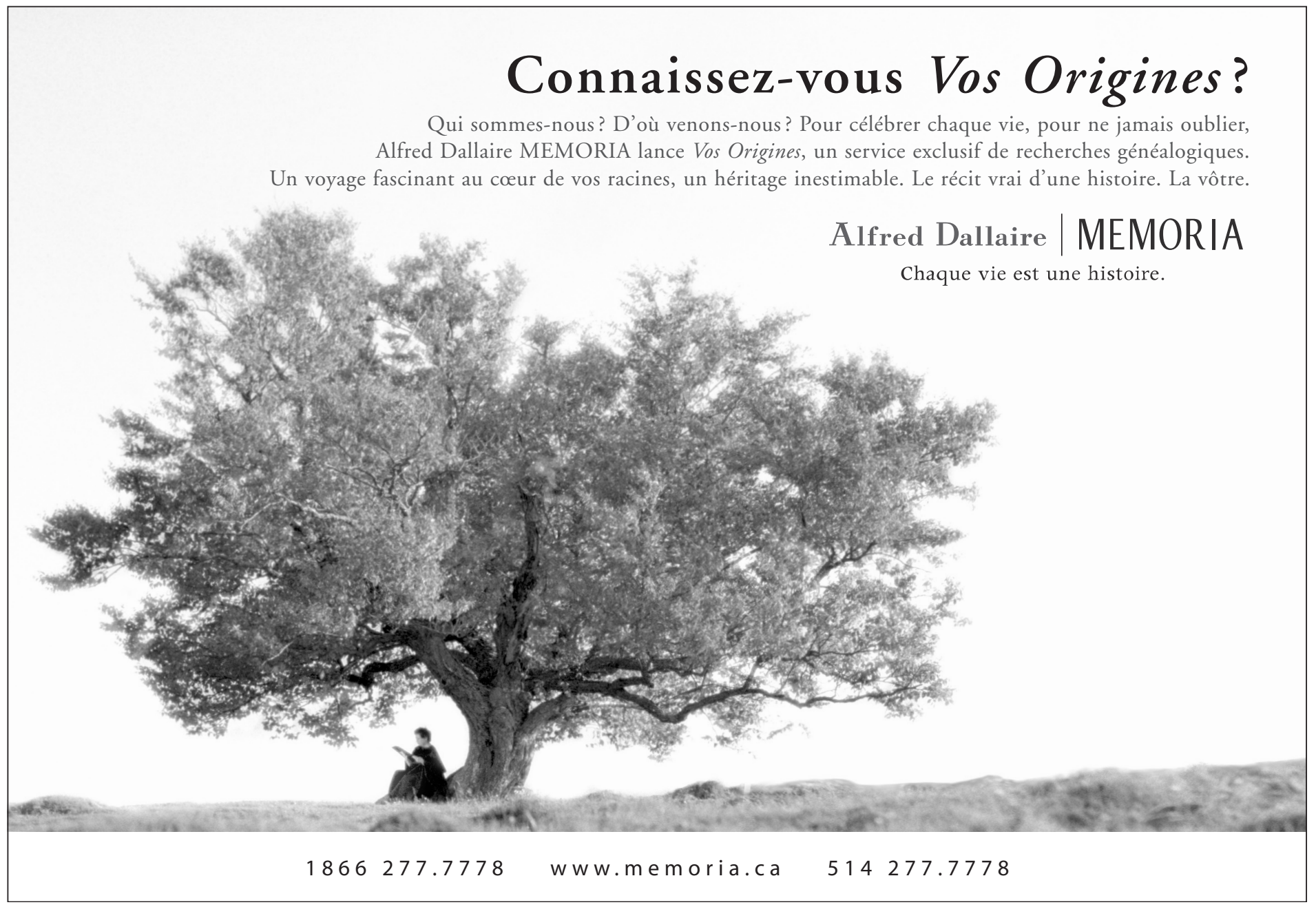

